\title{
Synergistic impact of Lactobacillus fermentum, Lactobacillus plantarum and vincristine on 1,2-dimethylhydrazine-induced colorectal carcinogenesis in mice
}

\author{
ASHA and DEVARAJA GAYATHRI \\ Department of Microbiology, Davanagere University, Davanagere 577002, Karnataka, India \\ Received January 6, 2012; Accepted March 15, 2012
}

DOI: $10.3892 /$ etm.2012.536

\begin{abstract}
Lactobacillus sp. is the most dominant probiotic strain of bacteria. Evidence indicates that the consumption of Lactobacillus sp. reduces the risk of colorectal cancer in animal models. The present study was carried out to determine whether administration of Lactobacillus fermentum/ Lactobacillus plantarum alone or in combination with vincristine have a synergistic impact on the control of colorectal cancer in an animal model. Mice with 1,2 dimethylhydrazine (DMH) hydrochloride-induced colon cancer were fed with L.fermentum and L. plantarum isolated along with vincristine. An increase in body weight, a decrease in ammonia concentration, a decrease in $\beta$ glucosidase and $\beta$ glucuronidase enzyme activity and a reduction in the number of crypts in the mice in the pre-carcinogen-induced group was noted when compared to these variables in the post-carcinogen-induced group. The body weight of the mice fed L.fermentum along with vincristine was increased $(6.5 \mathrm{~g})$, and was found to be 3.5 times higher compared to that of the control. A marked decrease in the ammonia concentration $(240 \mathrm{mg})$, and $\beta$ glucosidase (0.0023 IU) and $\beta$ glucopyranose enzyme activity (0.0027 IU) was observed; $22.59 \%$ less ammonia concentration, $73.26 \%$ less $\beta$ glucosidase activity and $56.46 \%$ less $\beta$ glucuronidase enzyme activity was noted when compared to the control. A significant reduction in the number of aberrant crypt foci (ACF) (90\%) was observed when compared to the control. Maximum protection was observed in the mice fed the probiotics and vincristine prior to cancer induction. Among the different dietary combinations tested in the present study, $L$. fermentum and vincristine showed a more extensive reduction in ammonia concentration, $\beta$ glucosidase, $\beta$ glucuronidase activity and the number of ACF.
\end{abstract}

Correspondence to: Professor Devaraja Gayathri, Department of Microbiology, Davanagere University, Davanagere 577002, Karnataka, India

E-mail: gayathridevaraja@gmail.com

Key words: Lactobacillus fermentum, Lactobacillus plantarum, 1,2-dimethylhydrazine, colorectal cancer

\section{Introduction}

Probiotics are the live cultures of beneficial organisms that selectively stimulate the growth of native bacteria in the gut, thereby benefiting the host (1). Probiotics from the genera Bifidobacteria, Lactobacillus and yeast are the most beneficial for the host intestine (2). Probioticos have been reported to be beneficial in controlling inflammation, lactose intolerance and cholesterol and have been found to exhibit antitumor activity and stimulation of the immune response, and also prevent allergies and reduce the incidence of respiratory diseases (3). It has also been reported that probiotics aid in the prevention of all types of diarrhea, inflammatory bowel diseases, ulcerative colitis, Crohn's disease, pouchitis, irritable bowel syndrome, constipation, liver diseases, lung cancer, mammary cancer and colorectal cancer (4).

Colorectal cancer (CRC) is one of the most common types of cancer worldwide and the second leading cause of cancerrelated death in developing and developed countries (5). L. bulgaricus (LAB) has been found to inhibit colon cancer by altering the metabolic activities of intestinal microflora, by altering physiochemical conditions, by binding, degradation, and inhibiting promoters to carcinogenesis, by producing certain metabolites, such as bile acid, by producing antimutagenic metabolites, by enhancing the host immune compounds, or by enhancing the host immune response (6). Modulation of enzymes, such as $\beta$ glucosidase and $\beta$ glucopyranose by probiotics results in a reduction of ras $\mathrm{p}-21$ oncoprotein activity which aids in cancer prevention (7).

It has been found that the consumption of probiotics resulted in an increase in the daily weight gain, the egg production, shell weight, shell thickness and concentration of cholesterol in the serum and yolk of leghorn chickens (8). It has also been reported that the consumption of Lactobacillus sp. by newborn ducks (Anas platyrhynchos domestica) and chicks (Gallus gallus domesticus) resulted in weight gain. Furthermore, the positive effect of probiotics in bringing health benefits also depends on the doses. A significant increase in body weight and liver mass was noted after the second dose of Lactobacillus administration in newborn chicks (9). In IL-10 knockout mice, probiotic Lactobacilli prevented and reduced the prevalence of colon cancer and mucosal inflammatory activity (10). Antitumorigenic activity of L. rhamnosus and Bifidobacterium lactis administration on azoxymethane-induced colon cancer 
was found to decrease carcinogenesis in rats (11). In laboratory investigations, certain strains of LAB have demonstrated antimutagenic effects due to their ability to bind to heterocyclic amines, which are carcinogenic substances formed in cooked meat (12). Animal studies have demonstrated that several LAB strains protect against colon cancer in rodents, although human data are limited and conflicting. Most human trials have found that the strains tested may exert anticarcinogenic effects by decreasing the activity of the $\beta$ glucuronidase enzyme (7). Possible mechanisms by which probiotics prevent colon cancer include the lowering of the $\mathrm{pH}$ of the intestine, the modulation of enzymes, such as $\beta$ glucosidase, which convert pro-carcinogens to proximate carcinogens, the reduction in the expression of ras-P21 oncoprotein, production of sodium butyrate by fermenting lactose, which is a powerful inhibitor of growth and an inducer of phenotype differentiation and apoptosis (13).

The plant Catharanthus roseus has been used from ancient times in treating various types of cancer, and this use has been described in Ayurveda (14). Its leaf, flower and root extracts contains more than 400 alkaloids approved as antineoplastic agents to cure leukemia, Hodgkin's disease, malignant lymphoma, neuroblastoma, rhabdomyosarcoma and Wilm's tumor (15). One ton of its leaves yields $50 \mathrm{~g}$ of vincristine sulfate in pure form. Vincristine and vinblastin are two important alkaloids used in the treatment of several types of cancer. Junior et al (16) evaluated the effects of vincristine on the gastrointestinal motility of awake rats and found an increase in the colon weight of these rats.

The antitumor effect as a result of the synergistic cytotoxic effect of a combination of betulinic acid and vincristine on malignant melanoma cells has also been reported (17). Furthermore, administration of topotecan and vincristine for pediatric solid tumor xenografts significantly resulted in a synergistic effect in controlling nine different types of cell lines: four neuroblastomas, three brain tumors and two rhabdomyosarcomas (18). Although Lactobacillus and vincristine are antitumorigenic in nature, reports on their combination in controlling CRC are scarce. Therefore, in the present study we investigated the activity of vincristine either alone and/or in combination with L. fermentum and L. plantarum in 1,2- dimethylhydrazine (DMH)-induced CRC in female Swiss mice.

\section{Materials and methods}

Mice. Experiments were performed on female Swiss albino mice weighing 25-35 g. All procedures and animal treatments were conducted in accordance with the Guide for the Care and Use of Laboratory Animals (S.S. Medical College Hospital, Davangere, India; Institutional Ethics committee) and were approved by the Animal Ethics Committee.

The mice were anesthetized with ether and treated with vincristine, which was slowly injected intraperitoneously i.p. for 21 days. Experimental animals received an equivalent amount of saline according to their weight.

Vincristine sulphate. Vincristine sulfate (MP Bioneutricals, Solon, $\mathrm{OH}, \mathrm{USA}$ ) was diluted with $1 \mathrm{mg} / \mathrm{ml}$ of saline, and $1 \mu \mathrm{l}$ of this solution was administered to each experimental mouse regularly up to 21 days. Every experimental group was injected with a single dose. Group 10 mice (VCR + DMH + normal diet) received only a vincristine dose, one week prior to carcinogen induction. Group 11 mice $(\mathrm{DMH}+\mathrm{VCR}+$ normal diet) received a vincristine injection after 1 week of carcinogen induction. Other groups received a vincristine injection along with L. fermentum and L. plantarum feeding pre- or post-carcinogen induction (Table I).

Carcinogen. DMH was purchased from Sigma Aldrich (C.3050). The carcinogen $(0.1 \mathrm{mg} / \mathrm{kg})$ was dissolved in physiological saline and $0.1 \mu \mathrm{l}$ was injected i.p., twice a week except for Group 1 (normal diet Control 1) and Group 9 (normal diet + VCR; Control 3). The injection was administered as a single dose and the animals were held carefully and injected without causing injury or discomfort.

Animals and treatments. We used 2- to 4-week-old female Swiss albino mice. The animals were housed in plastic cages with steel tops, and maintained in a 12-h light/12-h dark cycle at $28 \pm 2{ }^{\circ} \mathrm{C}$ and humidity $62 \pm 2 \%$. After their arrival, the animals ( $n=17$ per group) were acclimatized for 1 week, during which they were fed with standard ground nut cake. All the experimental animals were fed an equal amount of the diet $(100 \mathrm{~g} / \mathrm{kg}$ w/w). The pre-carcinogen-induced groups were fed with the probiotic diet or vincristine 1 week prior to carcinogen induction. Post-carcinogen-induced groups were fed with the probiotic diet or vincristine 1 week after the induction of the carcinogen. All the dietary groups were fed with a normal diet, apart from the pre- and post-carcinogen groups for which vincristine was also administered, which served as Control Group 3.

Bacterial strains. L. fermentum and L.plantarum were isolated from curd sample from remote regions of Karnataka, India. The sample was serially diluted and plated on MRS media and incubation was carried out at $37^{\circ} \mathrm{C}$ for $24 \mathrm{~h}$. The colonies obtained on MRS media were identified by physiological, fermentation test and were further subjected to PCR and identified as L. fermentum and L. plantarum (19). Overnight cultures of L. fermentum and L. plantarum were centrifuged at $8,000 \mathrm{~g} / 10 \mathrm{~min} / \mathrm{twice}$. Cells $\left(2 \times 10^{8}\right)$ were suspended in $1 \mathrm{ml}$ of skimmed milk and $0.1 \mathrm{ml}$ of this suspension was fed to the experimental mice regularly, apart from Control 2 (Group 2; $\mathrm{DMH}+$ normal diet).

Diets. Control diet, designed as a normal diet, consisted of starch $320 \mathrm{~g} / \mathrm{kg}$, casein $150 \mathrm{~g} / \mathrm{kg}$, ground nut malt $200 \mathrm{~g} / \mathrm{kg}$, a vitamin mix $15 \mathrm{~g} / \mathrm{kg}$ and a mineral mix $23 \mathrm{~g} / \mathrm{kg}$. The compositions of the normal diet have been obtained from the Ministry of Animal Husbandry, Government of Karnataka, India.

Lactobacillus fermentum diet. The culture in skim milk was added to the normal diet to provide $2 \times 10^{8} \mathrm{cfu} / \mathrm{g}$ diet.

Lactobacillus plantarum diet. The culture in skim milk was added to the normal diet to provide $2 \times 10^{8} \mathrm{cfu} / \mathrm{g}$ diet.

Lactobacillus fermentum/L. plantarum diet. This diet contained L.fermentum in skim milk (50\%) and L. plantarum in skim milk (50\%) added to the normal diet. The diets were prepared each day before feeding.

Experimental design. A total of 17 female 2- to 4-week-old Swiss albino mice were obtained, and after optimizing the physiological conditions, the mice were divided into 
Table I. Percentage of the weight gain of the mice in the experimental groups.

\begin{tabular}{|c|c|c|c|}
\hline Groups & Feeding schedule & Change in body weight & Percentage of weight gain \\
\hline Group 1 & Normal diet (Control 1) & +2 & $6.00 \%$ \\
\hline Group 2 & $\mathrm{DMH}+$ normal diet (Control 2) & -2 & $-6.25 \%$ \\
\hline Group 3 & Lf3 + DMH + normal diet & +6 & $21.00 \%$ \\
\hline Group 4 & $\mathrm{DMH}+\mathrm{Lf} 3+$ normal diet & +2 & $7.14 \%$ \\
\hline Group 5 & $\mathrm{Lp} 1+\mathrm{DMH}+$ normal diet & +3 & $10.34 \%$ \\
\hline Group 6 & $\mathrm{DMH}+\mathrm{Lp} 1+$ normal diet & +2 & $6.89 \%$ \\
\hline Group 7 & $\mathrm{Lf} 3+\mathrm{Lp} 1+\mathrm{DMH}+$ normal diet & +3 & $13.33 \%$ \\
\hline Group 8 & $\mathrm{DMH}+\mathrm{Lf} 3$ + Lp1 + normal diet & +1 & $6.66 \%$ \\
\hline Group 9 & VCR + normal diet (Control 3) & +3 & $10.71 \%$ \\
\hline Group 10 & VCR + DMH + normal diet (Control 4) & +5 & $17.85 \%$ \\
\hline Group 11 & $\mathrm{DMH}+\mathrm{VCR}+$ normal diet (Control 5) & +3 & $10.71 \%$ \\
\hline Group 12 & Lf3 + VCR + DMH + normal diet & +6.5 & $23.21 \%$ \\
\hline Group 13 & $\mathrm{DMH}+\mathrm{Lf} 3+\mathrm{VCR}+$ normal diet & +3 & $10.71 \%$ \\
\hline Group 14 & Lp1 + VCR + DMH + normal diet & +4 & $13.79 \%$ \\
\hline Group 15 & $\mathrm{DMH}+\mathrm{Lp} 1+\mathrm{VCR}+$ normal diet & +3 & $10.34 \%$ \\
\hline Group 16 & $\mathrm{Lf} 3+\mathrm{Lp} 1+\mathrm{VCR}+\mathrm{DMH}+$ normal diet & +2 & $10.00 \%$ \\
\hline Group 17 & $\mathrm{DMH}+\mathrm{Lf} 3+\mathrm{Lp} 1+\mathrm{VCR}+$ normal diet & +1 & $3.33 \%$ \\
\hline
\end{tabular}

DMH, 1,2-dimethylhydrazine hydrochloride; Lf3, Lactobacillus fermentum; Lp1, Lactobacillus plantarum; VCR, vincristine.

17 groups and fed the experimental diets as described above. Pre-carcinogen-induced mice were administered the DMH dose i.p. twice a week prior to 1 week of the probiotic/vincristine diet. Post-carcinogen-induced mice were administered DMH i.p. after 1 week of probiotic/vincristine dose. Food intake and body weight were measured each day. The rats were sacrificed by ether anesthetic 21 days after the start of the experimental diets. The cecum was removed and frozen at $-20^{\circ} \mathrm{C}$ for subsequent enzyme assay. The colon was removed and fixed for histopathological examination.

Assessment of aberrant crypt foci $(A C F)$. Colon (distal cecum to rectum) was removed from the anesthetized (sacrificed) experimental mice and slit longitudinally. Each colon was placed on a piece of white card, mucosal surface uppermost and the surface was cleaned gently with $0.9 \%$ saline. The back of the card was sprayed with absolute ethanol and the card and its attached colon was placed in $10 \%$ buffered formalin for at least $24 \mathrm{~h}$ for fixation of the tissue. The tissues were processed, sectioned using standard tissue processing procedure $(0.2 \mu \mathrm{m})$ and stained with H\&E. The number of ACF was determined using a low-power stereo microscope (MS-224; Magnus, Olympus, India).

Measurement of $\beta$ glucosidase and $\beta$ glucuronidase activity in cecal content. Samples of cecal contents from mice were homogenized (anerobic condition) in $0.1 \mathrm{M}$ potassium phosphate buffer/pH 7.2 to provide $10 \%$ (w/w) suspensions. The suspensions, in screw-cap bottles, were incubated anerobically with P-nitrophenyl- $\beta$-D-glucopyranoside (ONDP) $(3 \mathrm{mM})$ or P-nitrophenyl- $\beta$-D-glucuronide to determine the concentration of the enzyme $\beta$ glucosidase and $\beta$ glucuronidase, respectively. Released product P-nitrophenol was measured spectrophotometrically at $420 \mathrm{~nm}(20)$.
Measurement of cecal ammonia concentration. Samples of the $10 \%$ cecal homogenates were assayed for ammonia content using phenol nitroprusside and alkaline hypochloride (19). Blue color was developed and was measured spectrophotometrically $(570 \mathrm{~nm})$. The ammonia concentration was calculated with reference to a standard curve of ammonium chloride.

\section{Results}

Food and probiotic intake and body weight gain. The food intake of mice was similar for all of the experimental diets, and ranged between 2.8 and $3.3 \mathrm{~g} / \mathrm{mice} / \mathrm{day}$. The daily probiotic dose was $2 \times 10^{8} \mathrm{cfu} / \mathrm{kg}$ body weight per day for $30 \mathrm{-g}$ mice. There was a significant difference between the dietary mouse groups in final body weight or in body weight gain during the experiment. A significant increase in body weight of $\sim 6.5 \mathrm{~g}$ was noted for the mice fed with a combination of vincristine and L. fermentum (Lf3 + VCR + DMH + normal diet; Group 12), whereas an increase in weight by $\sim 6 \mathrm{~g}$ was observed in the Lf3 $+\mathrm{DMH}+$ normal diet group (Group 4). There was a significant weight gain observed in other groups ranging between 1 and $6 \mathrm{~g}$. However, the pre-carcinogenic group showed a marked increase in body weight, while the post-carcinogenic group showed a relatively lower increase in body weight (Table I).

Effect of L. fermentum, L. plantarum and vincristine on ammonia and activity of $\beta$ glucosidase and $\beta$ glucuronidase in cecal content. The concentration of ammonia in the cecal contents of the mice was significantly decreased upon the administration of L. fermentum (250 mg, 19.36\%) or L. plantarum (270 mg, 12.19\%) or vincristine and combination of L. fermentum $(240 \mathrm{mg}, 22.59 \%)$, L. plantarum $(260 \mathrm{mg}$, $16.13 \%)$ and vincristine $(250 \mathrm{mg}, 19.36 \%)$ (Fig. 1). Similarly, the activity of the enzyme $\beta$ glucuronidase was significantly 


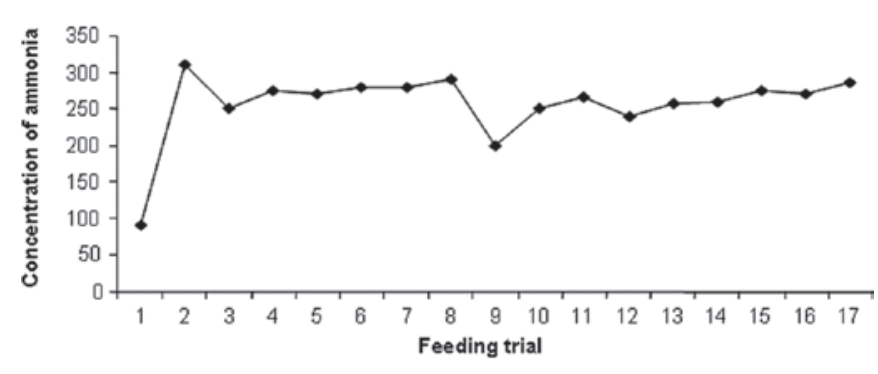

Ammonia concentration

Figure 1. Concentration of ammonia. 1, normal diet (Control 1); 2, normal diet + DMH (Control 2); 3, Lf3 + DMH + normal diet; 4, DMH + Lf3 + normal diet; 5, Lp1 + DMH + normal diet; 6, DMH + Lp1 + normal diet; 7, Lf3 + Lp1 + VCR + DMH + normal diet; 8, DMH + Lf3 + Lp1 + normal diet; 9, VCR + normal diet (Control 3); 10, VCR + DMH + normal diet (Control 4); 11, DMH + VCR + normal diet (Control 5); 12, Lf3 + VCR + DMH + normal diet; 13, DMH + Lf3 + VCR + normal diet; 14, Lp1 + VCR + DMH + normal diet 15, DMH + Lp1 + VCR + normal diet; 16, Lf3 + Lp1 + DMH + normal diet; $17, \mathrm{DMH}+\mathrm{Lf} 3+\mathrm{Lp} 1+\mathrm{VCR}+$ normal diet.

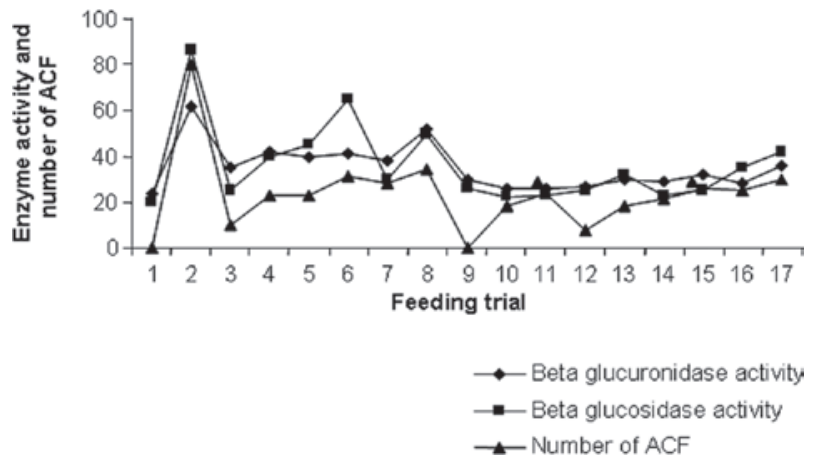

Figure 2. Enzyme activity of $\beta$ glucuronidase, $\beta$ glucosidase and number of ACF in experimental mice. 1, normal diet (Control 1); 2, normal diet + DMH (Control 2); 3, Lf3 + DMH + normal diet; 4, DMH + Lf3 + normal diet; 5, Lp1 + DMH + normal diet; 6, DMH + Lp1 + normal diet; 7, Lf3 + Lp1 + VCR + $\mathrm{DMH}+$ normal diet; 8, DMH + Lf3 + Lp1 + normal diet; 9, VCR + normal $\operatorname{diet}($ Control 3); 10, VCR + DMH + normal diet (Control 4); 11, DMH + VCR + normal diet (Control 5); 12, Lf3 + VCR + DMH + normal diet; 13, DMH + $\mathrm{Lf3}+\mathrm{VCR}+$ normal diet; 14, Lp1 + VCR + DMH + normal diet; 15, DMH + $\mathrm{Lp} 1+\mathrm{VCR}+$ normal diet; 16, Lf3 + Lp1 + DMH + normal diet; 17, DMH + $\mathrm{Lf} 3+\mathrm{Lp} 1+\mathrm{VCR}+$ normal diet.

decreased in all of the experimental groups in comparison to the mice fed the control diet (normal diet $+\mathrm{DMH}$; Control 2) (Fig. 2). Concentrations of ammonia and $\beta$ glucuronidase activity $(0.0027 \mathrm{IU}, 56.46 \%)$ were lowest in the mice fed vincristine and L. fermentum (Lf3 + VCR + DMH + normal diet; Group 12) (240 mg, 22.59\%) (Fig. 2). A maximum decrease in $\beta$ glucosidase enzyme activity (0.0023 IU, 73.6\%) was recorded in mice fed the combination of L.plantarum (Lp1 + VCR + DMH + normal diet; Group 14) (Fig. 2). Animals fed with $L$. fermentum alone exhibited slightly smaller $(250 \mathrm{mg}$, $19.36 \%$ ) reduction in ammonia concentration (0.0035 IU, $43.55 \%$ ), a relatively less decrease in $\beta$ glucuronidase activity and a smaller decrease $(0.0025$ IU, 70.4\%) in $\beta$ glucosidase enzyme activity. A significant reduction in ammonia concentration, $\beta$ glucuronidase activity and $\beta$ glucosidase enzyme activity was observed only in the pre-carcinogen-induced

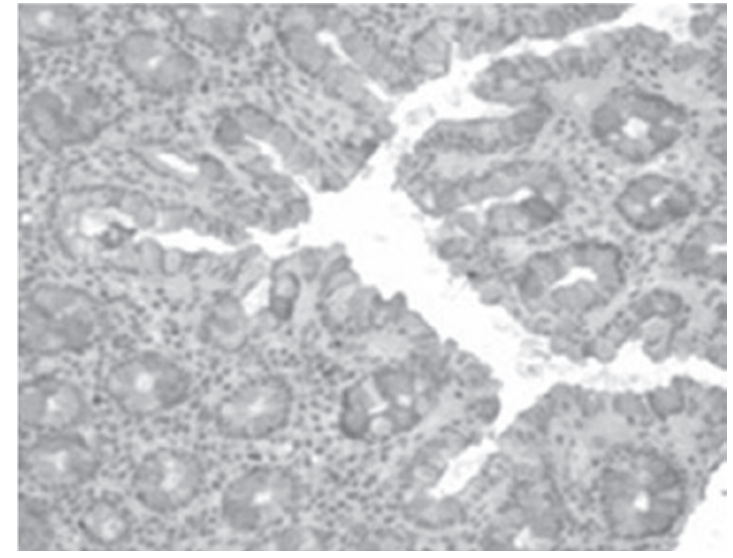

Figure 3. Normal colonic cells at low magnification (x10) in the control group.

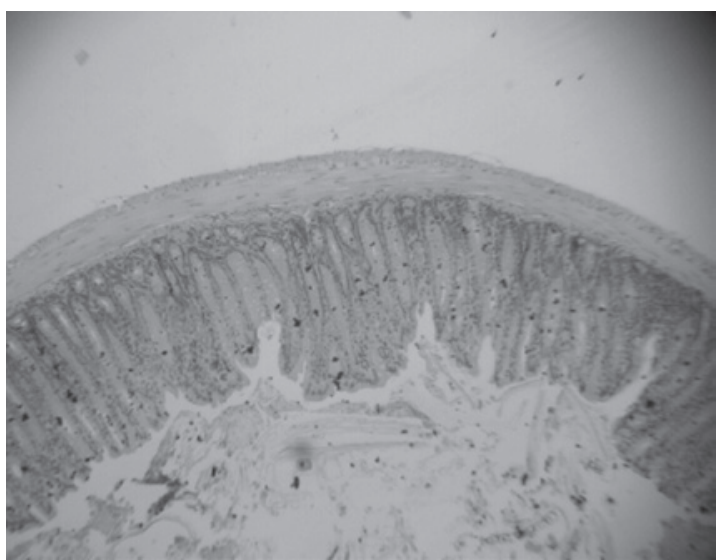

Figure 4. Enlarged colonic cell without the crypt at low magnification (x10) in the Lf3 + VCR + DMH + normal diet group (Group 12).

probiotic groups, while a smaller decrease was observed in the post-carcinogen-induced probiotic groups.

Effect of L. fermentum, L. plantarum and vincristine on ACF induction by 1,2-dimethylhydrazine dihydrochloride. In the DMH-treated mice, induced ACF were mainly distributed at the distal part of the colon. The number of aberrant crypts per focus was significantly decreased by 10 (87.5\%), $23(71.25 \%)$ and $18(77.5 \%)$ in the Lf3 + DMH + normal diet, $\mathrm{Lp1}+\mathrm{DMH}$ + normal diet and VCR + DMH + normal diet subgroups, respectively, in the pre-carcinogen-induced groups. However, the decrease in ACF was not as significant in the post-carcinogen-induced groups (Figs. 3-5). No ACF were detected in mice fed a normal diet in the control group that was not treated with a carcinogen.

The most observable inhibitory effect on DMH-induced $\mathrm{ACF}$ was found in the mice treated with the combination of L. fermentum and vincristine ( $\mathrm{Lf} 3+\mathrm{VCR}+\mathrm{DMH}+$ normal diet; Group 12), which decreased the total ACF number by (8 ACF) $90 \%$ in the pre-carcinogen-induced group (Fig. 4). However, there was no significant reduction in the numbers of ACF in the animals treated with L. fermentum, L. plantarum and vincristine in the post-carcinogen-induced groups. 


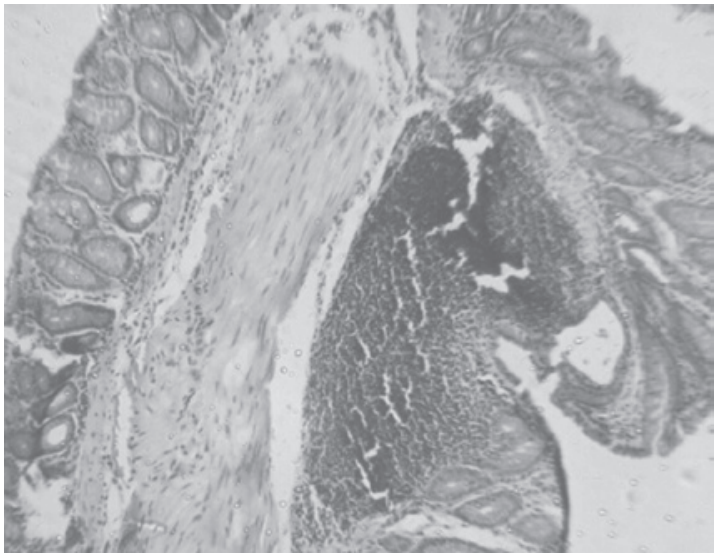

Figure 5. Larger tumor at low magnification (x10) in the normal diet $+\mathrm{DMH}$ group (Control 2).

\section{Discussion}

The present study was designed to assess the potential of $L$. fermentum and L. plantarum in preventing colorectal cancer and to study their synergistic impact in combination with a conventional chemotherapeutic drug vincristine. The Lactobacillus strains were isolated from remote regions of Karnataka, India, where individuals are regular consumers of curd, exhibiting longevity and disease endurance to gastrointestinal disorders.

Swiss albino mice (2- to 4-weeks of age) were administered DMH to produce CRC. The food intake was uniform, although there was a significant difference in the different experimental conditions. There was a marked weight gain in the experimental animals fed Lf3 in the pre-cancer induction group. Lactobacillus fermentum alone increased the body weight to approximately $6 \mathrm{~g}(21 \%)$, while an increase to $6.5 \mathrm{~g}$ (23.21\%) was observed in group 12 fed a combination of Lf3 + $\mathrm{VCR}+\mathrm{DMH}+$ normal diet. A $14 \%$ weight gain was previously noted when L. fermentum AD1 was administered for 4 days to 2-day-old Japanese quill Coturnix coturnix japonica (21), and the weight gain was $0.39 \mathrm{~g}$ more than that of the control group. A 23\% increase upon administration of Lf3 + VCR in the pre-cancerous group perhaps indicates the colonization of Lf3 in the intestine. A significant increase in $\operatorname{IgG}$ concentration and body weight was previously noted in 3- to 4-day-old male calves fed with $L$. acidophilus and L. plantarum containing a basal diet during the 5th week (22). Although a more rapid increase in body weight in Lactobacillus sp.-fed female broiler chicks (Gallus gallus domesticus) and ducks (Platyrhychos domestica) has been observed after the second dose (9), in the present study, a marked increase in body weight was recorded after the first dose. Intravenous vincristine treatment delayed gastric emptying and gastrointestinal motility transit of liquid in awake rats and an increase in colonic weight was also reported (20). Therefore, in the present study the synergistic effect of vincristine and the Lactobacillus isolates was evident; particularly Lf3 was potent in increasing body weight.

Ammonia concentration was significantly decreased in the cecal contents of the mice fed $L$. fermentum/vincristine (250 mg, 19.36\%) and L. plantarum (270 mg, 12.19\%). Mice fed a combination of L. fermentum and vincristine (Lf3 +
$\mathrm{VCR}+\mathrm{DMH}+$ normal diet; Group 12) showed a maximum reduction in ammonia concentration $(240 \mathrm{mg}, 22.59 \%)$. The other experimental groups of mice did not show a significant reduction in ammonia concentration, while in the cecal contents of rats a significant decrease by $25-30 \%$ was previously reported after administration of inulin or B. longum or both (20). Although $\beta$ glucuronidase activity was found to be decreased in L.fermentum (0.0035 IU, $43.55 \%)$, L. plantarum (0.0040 IU, 35.49\%) and vincristine (0.0030 IU, 54\%)-fed groups, a maximum decrease in $\beta$ glucuronidase activity was found in the mice fed a combination of L. fermentum and vincristine $[\mathrm{Lf} 3+\mathrm{VCR}+\mathrm{DMH}+$ normal diet $(0.0027 \mathrm{IU}$, $56.46 \%)]$. However, $\beta$ glucuronidase activity was previously found to decrease to $55 \%$ in rats fed inulin $+B$. longum (20), which perhaps indicates the potential of $\mathrm{Lp} 1$ in addition to Lf3 isolate.

$\beta$ glucosidase activity was found to decrease in the L. fermentum (0.0025 IU, 70.4\%), L. plantarum (0.0045 IU, 46.68\%) and vincristine (0.0025 IU, 70.94\%)-fed group. A maximum decrease in $\beta$ glucosidase enzyme activity was found in mice fed a combination of L. plantarum and vincristine $[\mathrm{Lp} 1+\mathrm{VCR}+\mathrm{DMH}+$ normal diet; Group 14 $(0.0023 \mathrm{IU}, 73.26 \%)]$. By contrast, a 7-fold increase in rats fet inulin $+B$. longumcompared to control rats was documented (20). Therefore, the fact that Lp1 and vincristine combination decreased the $\beta$ glucosidase activity perhaps indicates the novelty of this finding.

In the present study, L. fermentum (10 number of ACF, 87.5\%), L. plantarum (23 number of ACF, $71.25 \%$ ) and vincristine (18 number of ACF, 77.5\%)-fed mice showed a decreased $\mathrm{ACF}$ in DMH-induced carcinogenesis. A maximum reduction in ACF (8 number of crypts, 90\%) was found in the mice fed a combination of L. fermentum and vincristine [Lf3 + VCR + $\mathrm{DMH}+$ normal diet] and with DMH-induced carcinogenesis. Bifidobacterium longum and inulin were found to decrease the number of ACF in rats injected with AOM by 29 and 21\%, respectively, while the combination of inulin with $B$. longum decreased the total ACF by $74 \%$ (20). Although the combination of L. rhamnosus and B. lactis along with oligofructose inulin decreased the number of tumors in AOM-induced 4to 5-week-old male rats (11), the extent of decrease was not reported. Furthermore, Lactobacillus salivarius was shown to be associated with a reduced prevalence of colon cancer and mucosal inflammatory activity (10). Vincristine in combination with betulinic acid was also found to inhibit the metastasis of lung tumor cells in C57BL/6 mice (17). Therefore, Lf3 isolate in combination with vincristine may be a potential molecular complex for combating CRC showing a 90\% reduction in the number ACF. Activity of $\beta$ glucuronidase and the cecal ammonia concentration appeared to follow a similar pattern as the inhibition of ACF. These results suggest that the potential effects of Lp1/Lf3/vincristine feeding would be beneficial only when it is fed prior to induction of CRC carcinogen. However, the combination treatment strongly inhibited the number of ACF and the effect appeared to be more than additive. Administration of L. fermentum, L. plantarum or vincristine decreased the incidence in the colon of ACF, and combined treatment achieved a level of inhibition of $90 \%$, providing convincing evidence for a symbiotic effect. 


\section{Acknowledgements}

The authors thank the S.S. Institute of Medical College Hospital, Davanagere, India, for providing the animal laboratory facilities to conduct the experiments.

\section{References}

1. Jacela JY, DeRouchey JM, Tokach MD, et al: Feed additives for Swine: fact sheets - prebiotics and probiotics, and phytogenetics. J Swine Health Prod 18: 132-136, 2010.

2. Tomasik PJ and Tomasik P: Probiotics and prebiotics. Cereal Chem 80: 113-117, 2003.

3. Vanderhoof JA: Probiotics: future directions. Am J Clin Nutr 73 : 1152-1155, 2001.

4. Harish K and Varghese T: Probiotics in humans - evidence based review. Calicut Med J 4: 211-223, 2006.

5. Jiang $T$ and Savaiano DA: In vitro lactose fermentation by human colonic bacteria is modified by Lactobacillus acidophilus supplementation. J Nutr 127: 1489-1495, 1997.

6. Rafter J: Lactic acid bacteria and cancer: mechanistic perspective. Br J Nutr 88: 89-94, 2002.

7. Brady LJ, Gallaher DD and Busta FF: The role of probiotic cultures in the prevention of colon cancer. J Nutr 130: 410-414, 2000.

8. Panda AK, Reddy MR, Rao RSV, et al: Production performance, serum/yolk cholesterol and immune competence of white leghorn layers as influenced by dietary supplementation with probiotic. Trop Anim Health Prod 35: 85-94, 2003.

9. Angelakis E and Raoult D: The increase of Lactobacillus species in the gut flora of new born broiler chicks and ducks is associated with weight gain. PLoS One 5: 1-5, 2010.

10. Mahony LO, Feeney M, Halloran SO, et al: Probiotic impact on microbial flora, inflammation and tumour development in IL-10 knockout mice. Aliment Pharmacol Ther 15: 1219-1225, 2001.
11. Femia AP, Luceri C, Dolara P, et al: Antitumorigenic activity of the prebiotic inulin enriched with oligofructose in combination with the probiotic Lactobacillus rhamnosus and Bifidobacterium lactis on azoxymethane-induced colon carcinogenesis in rats. Carcinogenesis 23: 1953-1960, 2002.

12. Wollowski I, Rechkemmer G and Pool-Zobel BL: Protective role of probiotics and prebiotics in colon cancer. Am J Clin Nutr 73: 451-455, 2001

13. Jung JJ, Jeung HC, Lee JO, et al: Putative chemosensitive genes in colorectal cancer cell lines for anticancer agents. Oncol Rep 18: 593-599, 2007.

14. Garodia P, Ichikawa H, Malani N, et al: From ancient medicine to modern medicine: ayurvedic concepts of health and their role in inflammation and cancer. J Soc Integr Oncol 5: 1-14, 2007.

15. Nayak BS and Pereira LMP: Catharanthus roseus flower extract has wound healing activity in Sprague Dawley rats. BMC Complement Altern Med 6: 1-6, 2006.

16. Junior AAP, Teles BCV, Castro EFB, et al: Vincristine delays gastric emptying and gastro intestinal transit of liquids in awake rats. Braz J Med Biol Res 42: 567-573, 2009.

17. Sawada N, Kataoka K, Kondo K, et al: Betulinic acid augments the inhibitory effects of vincristine on grow th and lung metastasis of B16F10 melanoma cells in mice. Br J Cancer 90: 1672-1678, 2004.

18. Thompson J, George EO, Poquette CA, et al: Synergy of topotecan in combination with vincristine for treatment of pediatric solid tumor xenografts. Clin Cancer Res 5: 3617-3631, 1999.

19. Asha, Gayathri D and Batish V: Molecular characterization and variation of Lactobacillus sp. of remote malnad regions of Karnataka, India. Adv Environ Bio 6: 481-486, 2012.

20. RowlandIR, Rumney CJ,Coutts JT, et al:Effect of Bifidobacterium longum and inulin on gut bacterial metabolism and carcinogeninduced aberrant crypt foci in rats. Carcinogenesis 19: 281-285, 1998.

21. Strompfova V, Marcinakova M, Gancarcikova S, et al: New probiotic strain Lactobacillus fermentum AD1 and its effect in Japanese quail. Vet Med Czech 50: 415-420, 2005. 